\title{
Antiretroviral treatment uptake in patients with HIV- associated TB attending co-located TB and ART services
}

\author{
M D Nglazi, R Kaplan, J Caldwell, N Peton, S D Lawn, R Wood, L-G Bekker \\ The Desmond Tutu HIV Centre, Institute for Infectious Disease and Molecular Medicine, Faculty of Health Sciences, University of Cape Town \\ M D Nglazi, MPH \\ R Kaplan, MD \\ R Wood, MB BCh, MSc, FCP \\ L-G Bekker, MB ChB, FCP, PhD \\ S D Lawn, MD
}

International Union against Tuberculosis and Lung Disease, Paris, France

M D Nglazi, MPH

Department of City Health, City of Cape Town

J Caldwell, RN, RM, BCur

Provincial Government of the Western Cape, Cape Town

N Peton, MPA

Department of Infectious and Tropical Diseases, London School of Hygiene \& Tropical Medicine, London, UK

S D Lawn, MD

Corresponding author: M Nglazi (Mweete.Nglazi@hiv-research.org.za)

\begin{abstract}
Background. Delivery of integrated care for patients with HIV-associated TB is challenging. We assessed the uptake and timing of antiretroviral treatment (ART) among eligible patients attending a primary care service with co-located ART and TB clinics.

Methods. In a retrospective cohort study, all HIV-associated TB patients ( $\geq 18$ years old) who commenced TB treatment in 2010 were included. Data were analysed using basic descriptive statistics and log-binomial regression analysis.

Results. Of a total of 497 patients diagnosed with HIV-associated TB, 274 were eligible to start ART for the first time (median CD4 count, $159 \mathrm{cells} / \mu \mathrm{l})$. ART was started during TB treatment by 220 (80.3\%) patients. Among the 54 (19.7\%) who did not start ART, 23 (42.6\%) were either lost to follow-up (LTFU) or died before enrolling for ART; 12 (22.2\%) were either LTFU or died after enrolling but before starting ART; 5 (9.3\%) were transferred out; and 14 (25.9\%) only started ART after completion of TB treatment. The median delay between starting TB treatment and starting ART was 51 days (IQR 29 - 77). Overall, only 58.6\% of patients started ART within 8 weeks of TB treatment, and $12.7 \%$ of those with CD4 counts $<50$ cells/ $\mu \mathrm{l}$ started ART within 2 weeks.

Conclusions. In a setting with co-located TB and ART clinics, delays to starting ART were substantial, and one-fifth of eligible patients did not start ART during TB treatment. Co-location of services alone is insufficient to permit timely initiation of ART; further measures need to be implemented to facilitate integrated treatment.
\end{abstract}

S Afr Med J 2012;102(12):936-939. DOI:10.7196/SAMJ.6024

South Africa has a high burden of both TB and HIV ${ }^{1-3}$ and a high HIVassociated TB case fatality rate. ${ }^{4}$ Optimal case management of HIVassociated TB requires standardised anti-TB treatment combined with trimethoprim-sulphamethoxazole (co-trimoxazole) prophylaxis and antiretroviral treatment (ART). ART results in a $64-95 \%$ reduction in mortality risk ${ }^{5}$ and is an essential component of care. How soon to start ART after TB treatment initiation has become clearer from randomised controlled trials. These show that integration of ART and TB treatment in all HIV-associated TB patients regardless of CD4 count significantly improves survival, ${ }^{6}$ and that early ART (within 2 weeks of starting TB treatment) was particularly beneficial for patients with $\mathrm{CD} 4$ counts $<50$ cells/ $\mu 1 .{ }^{7-9}$ These data support the World Health Organization (WHO) ART 2010 guidelines that recommend ART is started within $2-8$ weeks of starting TB treatment. ${ }^{10}$

Although the guidelines recommending the optimal time to start ART in patients with HIV-associated TB have been established, the delivery of integrated care for these patients remains challenging. Models of integrated care in resource-limited countries include: efficient referrals between separately located TB and ART clinics; provision of $\mathrm{TB}$ and ART services on the same premises but by different service teams; and fully combined services with provision of TB treatment and ART from the same staff. ${ }^{11,12}$ The goal is to provide streamlined care, minimise patient losses and ensure timely initiation of ART.

Few data exist on patient losses from care and delays in starting ART among HIV-associated TB patients at different stages in the cascade between HIV-associated TB diagnosis and ART initiation in a variety of models of integrated care. ${ }^{13-18}$ Delivery of care in South Africa has largely been based within completely separate TB and ART services. In a primary care setting in a Cape Town township, we piloted delivery of care using co-located services in the same facility. We conducted this retrospective observational cohort study to assess the uptake and timing of ART initiation and losses from the care pathway. 


\section{Methods \\ Setting}

The study was conducted at Nyanga Community Health Centre (CHC), Klipfontein health sub-district, Cape Town. An estimated 420000 people $^{19}$ live in this predominantly low-income urban community, which had an antenatal HIV-1 prevalence rate of $24 \%$ in $2009 .{ }^{20}$ This nurse-run, doctor-supported service has provided TB and ART care for patients since 2008. Here, TB treatment and ART is delivered by separate health authorities but located in the same building. Most ART-eligible co-infected patients receiving $\mathrm{TB}$ treatment at the $\mathrm{TB}$ clinic were referred to the ART clinic at the same site, and a minority were transferred-out to separate ART clinics in different localities in Cape Town.

The clinic staff at the TB service followed the South African National TB guidelines ${ }^{21}$ for TB case detection, notification and treatment. TB was treated using standardised rifampicin-based regimens of 6 months' duration for new TB cases and 8 months for retreatment cases. During the study period, the South African ART guidelines were revised: before April 2010, staff at both TB and ART services followed the 2004 South African ART guidelines which recommended that patients with CD 4 counts of $50-200$ cells/ $\mu$ l should delay ART until after 2 months of starting TB treatment, while those with a CD4 count $<50$ cells/ $\mu$ or with serious co-morbidities should commence ART as soon as possible after at least 2 weeks of TB treatment. ${ }^{22}$ After April 2010, the clinic staff were advised to adopt the 2010 South African ART guidelines, which recommended that TB patients with CD4 cell counts $<100$ cells $/ \mu$ or WHO stage IV should commence ART within 2 weeks of eligibility, and those with CD4 cell counts $\leq 350$ cells/ $\mu$ should commence ART within 2 - 8 weeks of starting TB treatment. ${ }^{23}$

\section{Data sources}

The following data sources were used: (i) Western Cape's electronic eKapa TB and ART database which is currently being used at this clinic. TB data in this database have been prospectively maintained since October 2009, including demographic and clinical information on TB patients and on TB treatment visits and outcomes per TB episode. HIV data have been prospectively maintained since March 2007, including demographic data and clinical data on patients' ART treatment visits and outcomes. Both HIV and TB episodes are linked to patients using a unique identifier; (ii) paper-based TB register and the Western Cape's electronic TB register (ETR.net) which monitors TB treatment; (iii) National Health Laboratory Services (NHLS) database; and (iv) patient records.

\section{Data collection}

All HIV-associated TB patients $\geq 18$ years old who were registered and commenced on TB treatment between January 2010 and December 2010 were included. The Western Cape electronic data collection system, eKapa was used to obtain information on demographic characteristics (age and gender), clinical characteristics (TB disease classification, CD4 count and patient category) and TB treatmentrelated variables (date of starting TB treatment and regimen received). Information was also obtained on the uptake of ART and the time of ART initiation during TB treatment. Where information was missing, it was sought from patient records, NHLS database, paper based and electronic TB registers. Ethical clearance for the collection of routine data was obtained from the Research Ethics Committees of the University of Cape Town and the International Union against Tuberculosis and Lung Disease.

\section{Definitions}

Pre ART losses refer to failure to reach the next step in the care cascade from starting TB treatment to starting ART as a result of death and loss to follow-up (LTFU). Transfer to another TB and ART service was not regarded as a loss - patients who transfer are assumed to be retained.

Death refers to all-cause mortality.

Lost to follow-up refers to: (i) HIV-associated TB patients registered at the TB service who defaulted TB treatment for $\geq 8$ weeks and failed to enrol in the ART service; (ii) HIV-associated TB patients who enrolled in the ART service but failed to attend the ART service for $\geq 12$ weeks before starting ART; and (iii) patients classified as LTFU who were not known to have died or transferred to another facility.

ART eligibility was defined according to the South African national ART guidelines. ${ }^{22,23}$

\section{Statistical analyses}

Statistical analyses were performed using STATA statistical software, version 11.0 (STATA Corporation, College Station, Texas, USA). Basic descriptive statistics were used to characterise uptake of ART (using proportions) and timing of ART during TB treatment (using median and interquartile ranges). Proportions of patients who started ART within specific time points were calculated and stratified by CD4 count. The CD4 count strata were categorized as $<50$ cells $/ \mu \mathrm{l}$, and $50-200 \mathrm{cells} / \mu \mathrm{l}$ and $200-350 \mathrm{cells} / \mu \mathrm{l}$ according to the recent recommendations and guidelines. ${ }^{7-9,22,23}$ The time intervals considered were ART commencement between 2 and 8 weeks, and we determined the risk factors associated with starting ART after more than 8 weeks of TB treatment using log-binomial regression analysis.

\section{Results \\ Patient characteristics}

A total of 784 patients $\geq 18$ years started TB treatment from January - December 2010 (Fig. 1). Of these, 10 had unknown HIV status, 277 were HIV-negative and 497 were HIV-positive. Among the 497 patients diagnosed with HIV-associated TB, 130 were already receiving ART at the time of diagnosis (Fig. 1). Of the 367 not yet on ART at the time of TB diagnosis, 363 had CD4 cell count information available. Because the South African ART guidelines were revised during the study period, ART eligibility was assessed using CD4 cell counts $<200$ cells/ $\mu$ l prior to April 2010, and CD4 cells counts $\leq 350$ cells $/ \mu$ after April 2010. Overall, 274 were ART eligible and therefore formed the patient cohort of this study that was followed up to determine if ART was initiated appropriately during TB treatment.

The patient cohort $(N=274)$ characteristics at the time of start of TB treatment were: $51.8 \%$ of HIV-associated TB patients were female and of median age 34 years (interquartile range (IQR) 29 - 38) (Table 1). Immunodeficiency was advanced with a median blood CD4 lymphocyte count of 158.5 cells/ $\mu$ (IQR 69 - 303); 73.4\% co-infected patients had a new diagnosis of TB, and the majority of 194 (70.8\%) were diagnosed as pulmonary TB of whom $50.0 \%$ were confirmed smear-negative TB (99/194).

\section{Uptake of antiretroviral treatment}

Of the $274 \mathrm{HIV}$-associated TB patients who were eligible to start ART for the first time (median CD4 count, 158.5 cells/ $\mu l$ ), ART was started during TB treatment by 220 (80.3\%) patients; 54 (19.7\%) did not start ART (Fig. 1). Among the patients who did not start ART, 23 (42.6\%) were LTFU or died prior to enrolment for ART; 12 (22.2\%) were LTFU or died after enrolment but prior to starting ART; 5 (9.3\%) were transferred-out; and 14 (25.9\%) only started ART after completion of TB treatment. 
Table 1. Baseline characteristics for TB/HIV co-infected ARTeligible patients $\geq \mathbf{1 8}$ years old who started TB treatment in 2010

\begin{tabular}{|c|c|}
\hline Variables & $N=274$ \\
\hline \multicolumn{2}{|l|}{ Age, years } \\
\hline $18-35$ & $171(62.4)$ \\
\hline$\geq 36$ & $103(37.6)$ \\
\hline \multicolumn{2}{|l|}{ Gender } \\
\hline Female & $142(51.8)$ \\
\hline Male & $132(48.1)$ \\
\hline \multicolumn{2}{|l|}{ Patient category } \\
\hline New & $201(73.4)$ \\
\hline Retreatment & $73(26.6)$ \\
\hline \multicolumn{2}{|l|}{ Type of $\mathrm{TB}^{*}$} \\
\hline Smear-negative PTB & $99(36.1)$ \\
\hline Smear-positive PTB & $95(34.7)$ \\
\hline ЕРТВ & $80(29.2)$ \\
\hline \multicolumn{2}{|l|}{ CD4 count, cells/ $\mu \mathrm{l}$} \\
\hline$<50$ & $68(24.8)$ \\
\hline $50-200$ & $140(51.1)$ \\
\hline$>200$ & $66(24.1)$ \\
\hline \multicolumn{2}{|l|}{$\mathrm{TB}$ regimen } \\
\hline Regimen 1 & $200(73.0)$ \\
\hline Regimen 2 & $74(27.0)$ \\
\hline \multicolumn{2}{|c|}{$\begin{array}{l}\mathrm{PTB}=\text { pulmonary } \mathrm{TB} ; \mathrm{EPTB}=\text { extra-pulmonary } \mathrm{TB} \text {. } \\
{ }^{*} \text { Patients with no smears and having pulmonary TB were categorised as smear-negative } \\
\text { pulmonary TB. Patients having both pulmonary and extra-pulmonary TB were categorised } \\
\text { as having extra-pulmonary TB. }\end{array}$} \\
\hline
\end{tabular}

\section{Deaths}

Among the 54 patients who did not start ART, 15 (27.8\%). patients died; 13 died before enrolment for ART and 2 died after enrolment but prior to starting ART; 6 (40\%) of the 15 patients who died had CD4 cell counts $<50$ cells $/ \mu$ l.

\section{Time delays in starting antiretroviral treatment}

Among the 220 known to have started ART during TB treatment, 215 had ART initiation dates recorded in patient records. Of these, the overall median time delay between starting TB treatment and starting ART was was 51 days (IQR 29 - 77). Because of the survival benefit of starting within 2 weeks of TB treatment in patients with CD4 counts $<50$ cells/ $\mu \mathrm{l}$ and the national ART guideline reccommedation to start ART within 8 weeks for those with CD4 counts $<350$ cells/ $\mu$, we

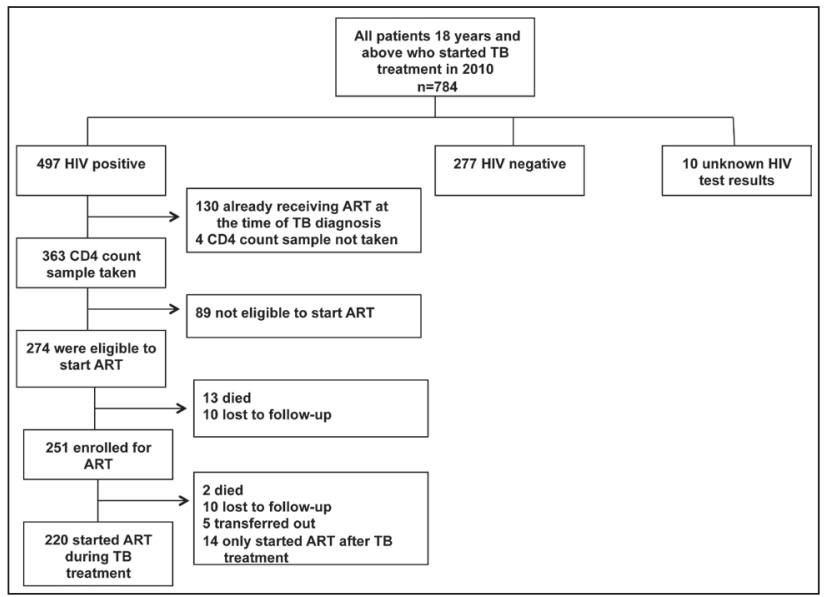

Fig. 1. Flow diagram showing uptake of ART treatment among HIVassociated TB patients. ART eligibility according to South African ART guidelines. ${ }^{22,23}$

examined delays in starting ART stratified by CD4 cell count. Among those with CD 4 counts $<50$ cells $/ \mu$ l, we found that only $12.7 \%(7 / 55)$ of them had started ART within 2 weeks (Table 2). In those with CD4 counts of 50 - 350 cells/ $\mu$ l, 53.1\% (85/160) started ART within 8 weeks.

\section{Risk factors associated with starting ART after 8 weeks of TB treatment}

We used univariate and multivariate analyses to determine the factors associated with starting ART after 8 weeks of TB treatment. Univariate analyses showed that patients with reccurrent TB were 2.4 times (95\% confidence interval (CI) 1.28 - 4.49) more likey to start ART after 8 weeks of TB treatment than those with new TB. The risk for starting ART after 8 weeks of TB treatment was higher among patients with CD4 cell counts of 50 - 200 cells $/ \mu$ (risk ratio (RR) 2.29; 95\% CI 1.12 4.66) and those with CD4 cell counts of 200 - 350 cells/ $\mu \mathrm{l}$ (RR 3.49; 95\% CI 1.51 - 8.07) compared with those with CD4 cell counts $<50$ cells $/ \mu$ l. Multivariate analyses showed similar results.

\section{Discussion}

In this study, we assessed the proportion of HIV-associated TB patients who successfully started ART and the timing of ART initiation during TB treatment. Of eligible patients, 19.7\% (54/274) did not start ART. However, there were losses in the cascade from ART eligibility to ART initiation. Of eligible patients who did not start ART, 42.6\% (23/54) were LTFU or died before enrolment for ART, and 22.2\% (12/54) were LTFU or died after enrolment but prior to starting ART. The delay in starting ART was substantial (51 days) and exceeded the recommendations of the 2010 WHO and national ART guidelines, ${ }^{10,23}$ especially for patients

Table 2. Duration of delays between starting TB treatment and starting ART among patients who initiated treatment with ART start date recorded and stratified by CD4 cell count category

\begin{tabular}{|c|c|c|c|c|}
\hline $\begin{array}{l}\text { Duration of delay between } \\
\text { starting TB treatment and } \\
\text { starting ART }\end{array}$ & $\begin{array}{l}\text { Patients with CD4 } \\
<50 \text { cells/ } \mu \mathrm{l}(n=55)\end{array}$ & $\begin{array}{l}\text { Patients with CD4 } \\
50-200 \text { cells } / \mu \mathrm{l}(n=114)\end{array}$ & $\begin{array}{l}\text { Patients with CD4 } \\
>200 \text { cells/ } \mu \mathrm{l}(n=46)\end{array}$ & All patients $(N=215)$ \\
\hline$<2$ weeks & $7(12.7)$ & $6(5.3)$ & $2(4.4)$ & $15(6.9)$ \\
\hline$<4$ weeks & $20(36.4)$ & 25 (21.9) & $8(17.4)$ & $53(24.7)$ \\
\hline$<6$ weeks & $30(54.6)$ & $46(40.4)$ & $11(23.9)$ & $87(40.5)$ \\
\hline$<8$ weeks & $41(74.6)$ & $64(56.1)$ & $21(45.7)$ & $126(58.6)$ \\
\hline$>8$ weeks & $14(25.5)$ & $50(43.9)$ & $25(54.4)$ & $89(41.4)$ \\
\hline
\end{tabular}


with CD4 cell counts $<50$ cells $/ \mu$ l. Therefore, despite co-locating TB and ART services, there are substantial delays in starting ART during TB treatment, and some patients did not start ART at all.

Our finding that $20 \%$ of eligible patients did not start ART lies within the range reported elsewhere in sub-Saharan Africa providing either separate or fully integrated services, of proportions of $13-86 \%,{ }^{13,14,16,17}$ which shows that there are barriers to successful uptake of ART regardless of the model of care applied, and further measures must be implemented to facilitate efficient treatment integration. Of those not starting ART, $42.6 \%$ had died or were LTFU before enrolment for ART, and 20\% of them died or were LTFU after enrolment but prior to starting ART. These data clearly indicate that retention in the stage in the cascade from ART eligibility to ART initiation must be improved. Retention might be improved by allowing ART services to dispense TB treatment and vice versa to streamline appointments and reduce duplication of services. Owing to the retrospective design of the study, the reasons for the reported losses are unknown. Possible reasons may include stigma, fear, lack of time to attend the clinic especially among those who work, and lack of transport money for additional clinic appointments. ${ }^{16,24}$ These factors might also have been induced by the presence of two health authorities rendering either $\mathrm{TB}$ or ART services that were not harmonised owing to organisational differences. Successful integration of TB and ART services may require integration at all levels in the health system from clinical care to community support and administrative structures.

We found an overall median delay in starting ART of 51 days, which is within the range of sub-Saharan Africa programmes providing either separate or fully integrated TB and ART services, of median delays between 44 and 110 days. ${ }^{14-17}$ While this median falls within the recommended 56 day cut-off, these patients have advanced HIV and more must be done within health systems to ensure earlier ART commencement. In particular, patients most at risk of AIDS mortality (CD4 counts $<50$ cells/ $\mu$ l) should be fast-tracked for ART; this may only be achieved if ART is prescribed and administered by the TB services.

The association between greater delays and higher CD4 cell counts is similar to a study ${ }^{16}$ among patients accessing separate TB and ART services in Cape Town, and is in keeping with the clinical recommendation that severely immunocompromised patients should be prioritised to initiate ART as soon as possible after starting TB treatment.

The strengths of this study include the tracking of losses at each stage in the cascade from start of TB treatment to start of ART, and time delays between starting TB treatment and starting ART in distinct services in this single primary health clinic. It provides an understanding of the strengths and limitations of an alternative model of TB and ART services and the importance of geographically co-locating these services, although not necessarily combining the services.

The study limitations are that there is no direct comparison with alternative models of either geographically separate or fully combined services; only one clinic with a relatively small sample size is considered, and the study does not quantify component delays associated with each step of the cascade from start of TB treatment to ART initiation. The retrospective design of the study does not enable us to determine the reasons for pre-ART losses among co-infected patients. Lastly, the uptake of ART among ineligible patients is unknown. Future studies will have to determine what happened to ART-ineligible patients.

\section{Conclusions}

ART services were included on the same premises that provided TB services at this primary health clinic in the hope of reducing delays in starting ART and improving ART uptake. However, this study found delays to starting ART were substantial, and a fifth of eligible patients did not start ART at all. These data show that the co-location of services alone is insufficient to permit timely initiation of ART, and further measures must be implemented to facilitate integrated treatment.

Acknowledgements. The authors gratefully acknowledge the dedicated staff of the Nyanga Community Health Centre. MDN is supported as an operational research fellow by the International Union Against Tuberculosis and Lung Disease, Paris, France. SDL is funded by the Wellcome Trust, London UK. RW is funded in part by the Cost-Effectiveness of Preventing AIDS Complications (CEPAC) funded by the National Institutes of Health (NIH, 5 R01AI058736-02), the International Epidemiologic Database to Evaluate Aids with a grant from the National Institute of Allergy and Infectious Diseases (NIAID: 5U01AI069924-02), USAID Right to Care (CA 674 A 0008 0000 700) and the South African Centre for Epidemiological Modelling and Analysis (SACEMA).

\section{References}

1. World Health Organization. Global HIV/AIDS response: Epidemic Update and Health Sector Progress toward Universal Access. Progress Report 2011. Geneva: World Health Organization, 2011. http://whqlibdoc.who.int publications/2011/9789241502986_eng.pdf (accessed 8 March 2012).

2. Lawn SD, Zumla AI. Tuberculosis. Lancet 2011;378(9785):57-72. [http://dx.doi.org/10.1016/S01406736(10)62173-3] [PMID:21420161]

World Health Organization. Global Tuberculosis Control. Geneva: World Health Organization, 2011. http:// www.who.int/tb/publications/global_report/2011/gtbr11_full.pdf (accessed 15 May 2012).

4. Bekker L-G, Wood R. TB and HIV co-infection: When to start antiretorviral therapy. S Afr J Contin Med Educ 2011;29(10):420-426.

Lawn SD, Kranzer K, Wood R. Antiretroviral therapy for control of the HIV-associated tuberculosis epidemic in resource-limited settings. Clin Chest Med 2009;30(4):685-699. [http://dx.doi.org/10.1016/.ccm.2009.08.010] [PMID:19925961] [PMCID:PMC2887494]

6. Abdool Karim SS, Naidoo K, Grobler A, et al. Timing of initiation of antiretroviral drugs during tuberculosis therapy. N Engl J Med 2010;362(8):697-706. [http://dx.doi.org/10.1056/NEJMoa0905848] [PMID: 20181971] [PMCID:PMC3076221]

Abdool Karim SS, Naidoo K, Padayatchi N, et al. Optimal timing of ART during TB therapy; findings of the SAPIT trial. 18th Conference on Retroviruses and Opportunistic Infections, Boston, MA, USA. Abstract 39LB. SAPI

2011.

Blanc FX, Sok T, Laureillard D, et al. Significant enhancement in survival with early (2 weeks) v. late (8 weeks) initiation of highly active antiretroviral treatment (HAART) in severely immunosuppressed HIV-infected adults with newly diagnosed tuberculosis. AIDS 2010 - XVIII International AIDS Conference, Austria, Vienna. Abstract no. THLBB106. 2010.

9. Havlir DV, Kendall MA, Ive P, et al. Timing of antiretroviral therapy for HIV-1 infection and tuberculosis N Engl J Med 2011;365(16):1482-1491. [http://dx.doi.org/10.1056/NEJMoa1013607] [PMID:22010914] [PMCID:PMC3327101]

10. World Health Organization. Antiretroviral Therapy for HIV Infection in Adults and Adolescents. Recommendations for a Public Health Approach (2010 revision). Geneva: World Health Organization, 2010. http://www.who.int/hiv/pub/arv/adult//en/index.html (accessed 14 February 2012).

11. World Health Organization. Priority Research Questions for Tuberculosis/Human Immunodeficiency Virus World Health Organization. Priority Research Questions for Tuberculosis/Human Immunodeficiency
(TB/HIV) in HIV-prevalent and Resource-limited Settings. Geneva: World Health Organization, 2010.

12. World Health Organization. WHO Policy on Collaborative TB/HIV Activities: Guidelines for National World Health Organization. WHO Policy on Collaborative TB/HIV Activities: Guidelines for National
Programmes and other Stakeholders. Geneva: World Health Organization, 2012. http://whqlibdoc.who.int/ Programmes and other Stakeholders. Geneva: World Health Orga
publications/2012/9789241503006_eng.pdf (accessed 22 May 2012).

publications/2012/9789241503006_eng.pdf (accessed 22 May 2012).
13. Zachariah R, Harries AD, Manzi M, et al. Acceptance of anti-retroviral therapy among patients infected with HIV and tuberculosis in rural Malawi is low and associated with cost of transport. PLoS One 2006;1(1):e121. [http://dx.doi.org/10.1371/journal.pone.0000121] [PMID:17205125][PMCID:PMC1762339]

14. Brown C, Kerschberger B, Boulle A, et al. TB and HIV service integration within a South African primary health care setting reduces the time to ART initiation without negatively impacting TB outcomes.18th Conference on Retroviruses and Opportunistic Infections, Boston, USA. Abstract 890. 2011.

15. Lawn SD, Campbell L, Kaplan R, et al. Time to initiation of antiretroviral therapy among patients with HIVassociated tuberculosis in Cape Town, South Africa. J Acquir Immune Defic Syndr 2011;57(2):136. [http:/ dx.doi.org/10.1097/QAI.0b013e3182199ee9] [PMID:21436714]

16. Lawn SD, Campbell L, Kaplan R, Little F, Morrow C, Wood R. Delays in starting antiretroviral therapy in patients with HIV-associated tuberculosis accessing non-integrated clinical services in a South African township. BMC Infect Dis 2011;11:258. [http://dx.doi.org/10.1186/1471-2334-11-258] [PMID:21957868] township. BMC Infect DiD.PMC3203070]

17. Pepper DJ, Marais S, Wilkinson RJ, Bhaijee F, De Azevedo V, Meintjes G. Barriers to initiation of antiretrovirals Pepper DJ, Marais S, Wilkinson RJ, Bhaijee F, De Azevedo V, Meintjes G. Barriers to initiation of antiretrovirals
during antituberculosis therapy in Africa. PLoS One. 2011;6(5):e19484.[ http://dx.doi.org/10.1371/journal. during antituberculosis therapy in Africa. PLoS One. 201
pone.0019484 ][ PMID:21589868] [PMCID:PMC3093394]

pone.0019484 ][ PMID:21589868] [PMCID:PMC3093394]
18. Hermans SM, Castelnuovo B, Kambugu A, Lange JMA, Hoepelman AIM, Manabe YC. Integration of HIV and TB services results in earlier and more prioritised ART initiation in Uganda. 6th IAS Conference on HIV Pathogenesis and Treatment, Rome, Italy. Abstract WEPDD0103. 2011.

19. Statistics South Africa Mid-year Population Estimates. Department of Health, South Africa. Pretoria: Statistic South Africa, 2010. http://www.statssa.gov.za/publications/P0302/P03022010.pdf (accessed 18 August 2011).

20. National Department of Health. 2009 National Antenatal Sentinel HIV and Syphilis Prevalence Survey, South Africa. Pretoria: National Department of Health, 2010.

21. National Department of Health.The South African National Tuberculosis Control Programme Practical Guidelines 2004. Pretoria: National Department of Health, 2004.

22. National Department of Health. National Antiretroviral Treatment Guidelines. Pinetown, South Africa: Jacan Publishers, 2004.

23. National Department of Health. Clinical Guidelines for the Management of HIV\&AIDS in Adults and Adolescents. Pretoria: National Department of Health, 2010.

24. Daftary A, Padayatchi N. Social constraints to TB/HIV healthcare: Accounts from coinfected patients in South Africa. AIDS Care 2012; 24 April. [http://dx.doi.org/10.1080/09540121.2012.672719] [PMID:22530855] 